\title{
miR-200a suppresses cell growth and migration by targeting MACC1 and predicts prognosis in hepatocellular carcinoma
}

\author{
JIYE FENG ${ }^{1}$, JINBO WANG ${ }^{1}$, MINGLIANG CHEN ${ }^{1}$, GUN CHEN ${ }^{1}$, ZONGYANG WU ${ }^{1}$, \\ LIPING YING $^{1}$, QIFENG ZHUO ${ }^{1}$, JIANLEI ZHANG ${ }^{1}$ and WEILIN WANG ${ }^{2}$
}

\author{
${ }^{1}$ Department of Hepatobiliary Surgery, Ningbo Yinzhou People's Hospital (Yinzhou Hospital Affiliated to \\ Medical School of Ningbo University), Ningbo, Zhejiang 315040; ${ }^{2}$ Division of Hepatobiliary and Pancreatic Surgery, \\ Department of Surgery, The First Affiliated Hospital, Zhejiang University School of Medicine, \\ Hangzhou, Zhejiang 310003, P.R. China
}

Received September 12, 2014; Accepted November 14, 2014

DOI: 10.3892/or.2014.3642

\begin{abstract}
R-200a suppresses tumor development and progression; however, its role in tumor growth and metastasis of hepatocellular carcinoma (HCC) and the underlying mechanism have not been elucidated. In the present study, we identified that miR-200a was markedly downregulated in HCC and exerted suppressive effects on tumor cell growth and metastasis. We identified that miR-200a suppressed tumor growth and metastasis by directly targeting MACC1. In addition, HCC patients with low miR-200a expression had significantly worse prognosis than those with high expression of miR-200a. These findings suggest that miR200a may be recognized as a novel potential biomarker to predict the survival of patients with HCCs following liver transplantation.
\end{abstract}

\section{Introduction}

Hepatocellular carcinoma (HCC) is a highly aggressive cancer, characterized by the activation of multiple molecular pathways (1-5). It is the sixth most common cancer and the third most common cause of cancer-related deaths worldwide (6-9). China alone accounts for $55 \%$ of the world's cases due to the high prevalence of chronic hepatitis B virus (HBV) infection and liver cirrhosis, both significant risk factors for the disease (10-13). Currently, radical resection and liver transplantation (LT) offer the main potentially curative therapeutic modalities. However, the long-term survival of patients

Correspondence to: Dr Jiye Feng, Department of Hepatobiliary Surgery, Ningbo Yinzhou People's Hospital (Yinzhou Hospital Affiliated to Medical School of Ningbo University), Ningbo, Zhejiang 315040, P.R. China

E-mail: fengjiye@163.com

Key words: miR-200a, MACC1, prognosis, hepatocellular carcinoma following surgery remains unsatisfactory due to the high frequency of recurrence due to metastasis - principally attributed to the presence of microscopic extrahepatic metastatic foci before surgery $(14,15)$. Therefore, a better understanding of the molecular mechanisms underlying $\mathrm{HCC}$ recurrence may offer improved diagnostic and prognostic capabilities in addition to the development of effective novel therapeutic strategies.

microRNAs, a newly discovered class of non-coding small RNAs, have been recognized as potential targets for tumor treatment due to their important roles in carcinogenesis and cancer progression via binding to specific complementary sites within the 3' untranslated regions (3'UTR) of their target gene mRNAs (16-18). Accumulated studies have shown that miRNAs may function as oncogenes or tumor suppressors in the tumorigenesis of various human cancers depending on the cellular contexts and the target genes that they regulate. Among these functional miRNAs, miR-200a has been demonstrated to function as a tumor suppressor, and loss of miR-200a expression has been reported in many cancer types, whereas restoration of miR-200a expression has been shown to abrogate tumorigenesis $(19,20)$. For instance, decreased miR-200a has been detected in breast cancer, whereas enhanced expression of miR-200a was found to induce growth suppression in breast cancer cells through downregulation of mitochondrial transcription factor A (TFAM) expression (21). Moreover, miR-200a was observed to inhibit gastric adenocarcinoma cell proliferation and migration by suppressing the wnt/ $\beta$-catenin pathway (22).

However, the role of miR-200a in cell survival and metastasis of liver cancer and the underlying molecular mechanisms have not been elucidated. We therefore aimed to ascertain whether miR-200a may also play an important role in the tumor progression and metastasis of HCC.

We investigated the gene expression pattern of miR-200a in HCC and analyzed its effects on facets of HCC biology including invasion and cell survival. By revealing the functional implications and molecular mechanisms of miR-200a, we shed crucial light on how miR-200a functions in HCC pathogenesis. 


\section{Materials and methods}

Tissue samples, cell lines and cell transfection. One hundred and fifteen pairs of HCC and their matched adjacent normal liver tissues were collected. The clinicopathological characteristics of the patients are shown in Table I. Specimens of cancer tissues and their adjacent noncancerous tissues and clinical information were available from these patients after obtaining informed consent. All samples were obtained from patients who underwent LT at Ningbo Yinzhou People's Hospital [Yinzhou Hospital Affiliated to the Medical School of Ningbo University and The First Affiliated Hospital of Zhejiang University School of Medicine (Hangzhou, China)]. All samples were snap-frozen in liquid nitrogen and then stored at $-80^{\circ} \mathrm{C}$ for further use. This study was approved by the Ethics Review Committee of Ningbo Yinzhou People's Hospital (Yinzhou Hospital Affiliated to the Medical School of Ningbo University and The First Affiliated Hospital, School of Medicine, Zhejiang University).

Eight human HCC cell lines, including HepG2, SMMC7721, BEL7402, Huh7, HCCLM3, MHCC97L, PLC and normal liver cell line LO2 were purchased from the American Type Culture Collection (Manassas, VA, USA), the Shanghai Institute of Cell Biology (Shanghai, China) and the Liver Cancer Institute of Fudan University (Shanghai, China). All of the cell lines were cultured in Dulbecco's modified Eagle's medium (DMEM) with $4.5 \mathrm{~g} / \mathrm{l}$ glucose (HyClone) supplemented with $10 \%$ fetal bovine serum (FBS) (SAFC Biosciences) and incubated at $37^{\circ} \mathrm{C}$ in a humidified environment containing $5 \% \mathrm{CO}_{2}$.

Ectopic expression of miR-200a in the cells was achieved by transfection with miR-200a mimics or inhibitors (Qiagen, Hilden, Germany) using Lipofectamine 2000 (Invitrogen, Carlsbad, CA, USA). Cells were plated in 6-well plates and transfected for 24 or $48 \mathrm{~h}$. Transfected cells were used in further assays or RNA/protein extraction.

RNA extraction and SYBR-Green quantitative PCR analysis. Total RNA was extracted from the cells using TRIzol reagent (Invitrogen). Mature miR-200a expression in the cells was detected using the miScript SYBR-Green PCR kit (Qiagen). Expression of RNU6B was used as an endogenous control. MACC1 expression was measured by SYBR-Green qPCR assay (Takara, Dalian, China). $\beta$-actin and glyceraldehyde 3-phosphate dehydrogenase (GAPDH) were used as internal controls and processed using the $2^{-\Delta \Delta \mathrm{Ct}}$ formula, and then normalized to the internal control. PCR of MACC1 was performed with specific primers: forward, TCGGTC AGGAAGAATTGCAC and reverse, TTGTGAAGCAAG TCTGGGTCC.

In situ hybridization for miRNA. In situ hybridization for miR-200a was performed in the formalin-fixed and paraffin-embedded tissue specimens by using the miRCURY LNA microRNA ISH Optimization kit (Exiqon, Vedbaek, Denmark). A 5'-DIG and 3'-DIG miRCURY labeled miR-200a LNA probe (cat. 18094-15; Exiqon) was used. A scrambled LNA detection probe was used as a negative control (cat. 99004-01; Exiqon). Briefly, $5 \mu$ m-thick paraffin sections were deparaffinized and treated with proteinase- $\mathrm{K}$ $(15 \mu \mathrm{g} / \mathrm{ml})$ at $50^{\circ} \mathrm{C}$ for $60 \mathrm{~min}$, followed by stringent washes with $5 \mathrm{X}$ standard saline citrate, $1 \mathrm{X}$ saline sodium citrate, and $0.2 \mathrm{X}$ saline sodium citrate buffers at $50^{\circ} \mathrm{C}$; DIG blocking reagent (Roche, Mannheim, Germany) in maleic acid buffer containing $2 \%$ sheep serum at room temperature for $15 \mathrm{~min}$; and alkaline phosphatase-conjugated anti-digoxigenin (diluted 1:500 in blocking reagent; Roche) at room temperature for 60 min. Enzymatic development was performed by incubating the slides with 4-nitro-blue tetrazolium and 5-brom-4-chloro$3^{\prime}$-indolylphosphate substrate (Roche) at $30^{\circ} \mathrm{C}$ for $2 \mathrm{~h}$ at $37^{\circ} \mathrm{C}$ for $10 \mathrm{~min}$, followed by nuclear fast red counterstain (Sangon, Shanghai, China) at room temperature for $1 \mathrm{~min}$. Slides were then dismantled in water, dehydrated in alcohol solutions and mounted with mounting medium (Sangon).

Measurement of cell viability, colony formation assay, invasiveness and migration. Cell viability was measured using the Cell Counting Kit-8 (Dojindo Laboratories, Kumamoto, Japan). Cells $\left(0.2 \times 10^{4}\right)$ transfected with the indicated miRNA or siRNA for $24 \mathrm{~h}$ were seeded in each 96-well plate.

For the colony formation assay, cells were seeded at 200 cells/well in 6-well plates containing complete DMEM on day 0 , which was refreshed twice/week. On day 14 , colonies were fixed with $3.7 \%$ formaldehyde for $15 \mathrm{~min}$ and stained with $1 \%$ crystal violet (Sangon) before quantification.

Cell invasion and migration assays were performed using the Transwell (Millipore, Billerica, MA, USA) based method. Forty eight hours after plasmid transfection or RNA interference, the filters coated with Matrigel (BD Bioscience, San Jose, CA, USA) in the upper compartment were seeded with $0.4 \times 10^{5}$ or $0.8 \times 10^{5}$ cells. After 48 or $72 \mathrm{~h}$, invaded cells on the bottom surface were stained with $0.1 \%$ crystal violet (Sangon). The cell migration assay was performed similarly, except that the cells were applied to the uncoated filter.

Western blotting. Western blotting was used to detect the expression of target genes at the protein level. Protein was extracted from the transfected MHCCLM3 cells using modified RIPA buffer in the presence of proteinase inhibitor cocktail. Equivalent quantities (30-50 $\mu \mathrm{g})$ of protein were separated on $10 \%$ SDS-polyacrylamide gels and transferred to polyvinylidene difluoride membranes. The membranes were blocked with 5\% non-fat milk and then incubated overnight at $4^{\circ} \mathrm{C}$ with the appropriate primary antibody at the dilutions specified by the manufacturer. The membranes were then washed three times in $10 \mathrm{ml}$ TBST and incubated with the corresponding horseradish peroxidase (HRP)-conjugated secondary antibody at a 1:2,000 dilution for $1 \mathrm{~h}$. The bound secondary antibody was detected using an enhanced chemiluminescence (ECL) system (Pierce Biotechnology Inc., Rockford, IL, USA). Primary antibodies were as follows: MACC1 antibody (1:250) and anti- $\beta$-actin antibody $(1: 1,000)$ (both from Sigma, St. Louis, MO, USA). $\beta$-actin protein was used as the internal control.

miRNA target prediction. For the prediction of the target genes and the 3'UTR binding sites by the seed region of miR-200a, the TargetScan (http://www.targetscan.org/), miRanda (http:/www. microrna.org/microrna/home.do) and PicTar (http://pictar.mdc-berlin.de/) databases were used. The related functions of the targets were also considered. 


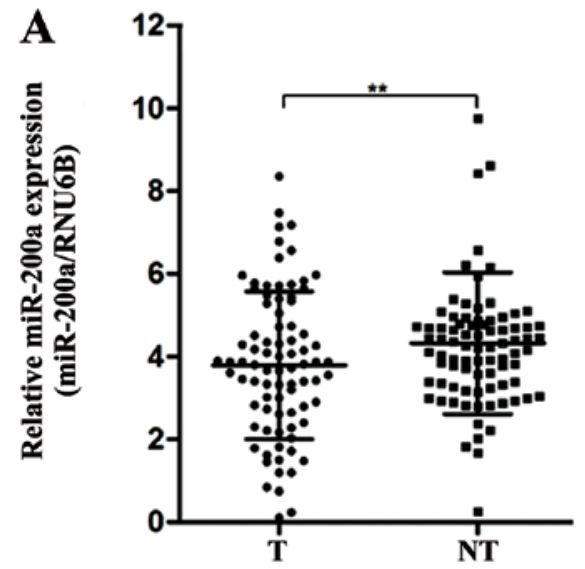

B The expression level of miR-200a (ISH)
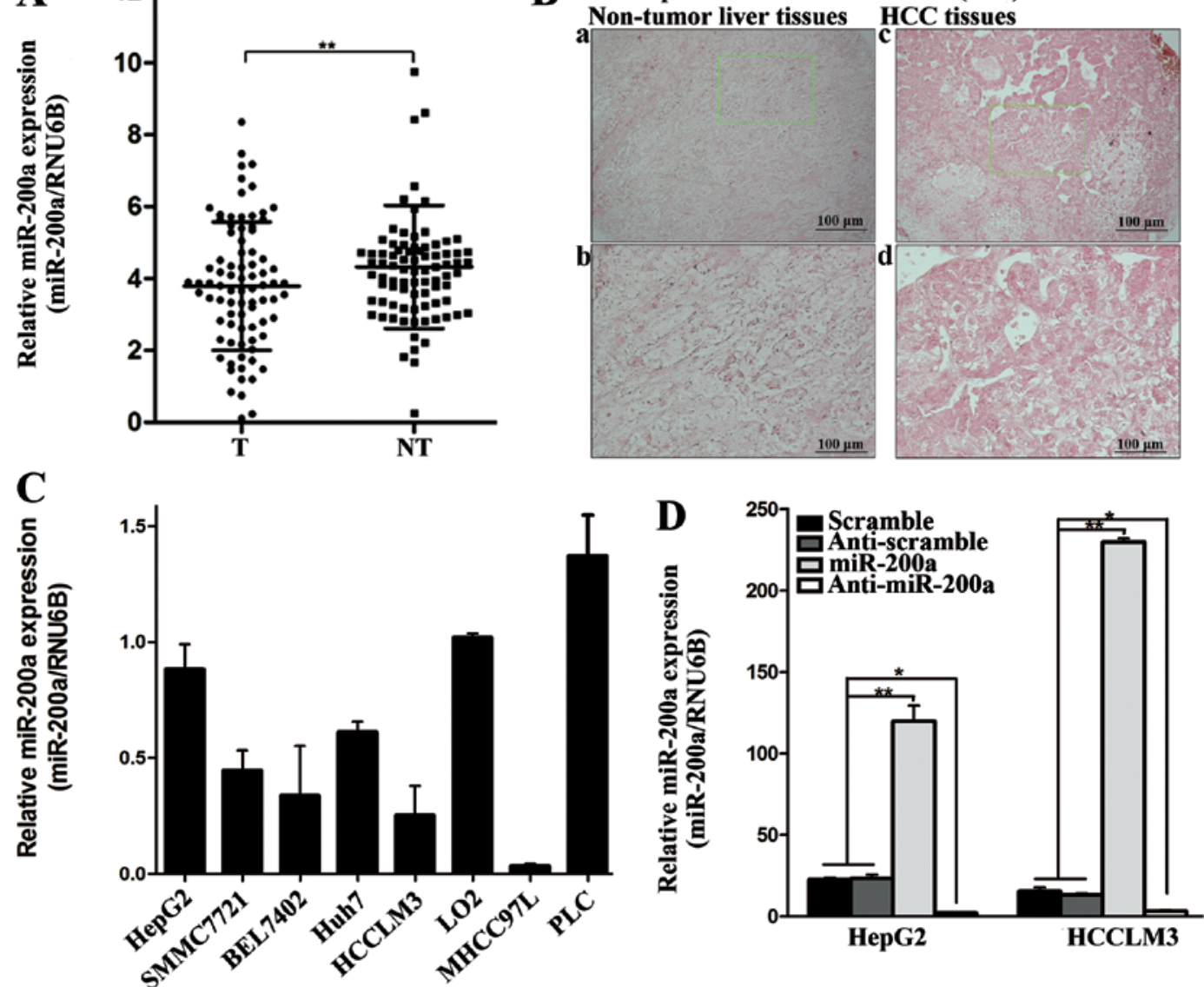

Figure 1. miR-200a is frequently downregulated in hepatocellular carcinoma (HCC) and inhibits tumor cell growth and metastasis in vitro. (A) Expression of miR-200a in 115 pairs of HCC tumor tissues (T) and the corresponding non-tumor (NT) liver tissues. Expression levels of miR-200a were normalized to the corresponding levels of RNU6B. Each sample was analyzed in triplicate, and values are expressed as levels (mean $\pm \mathrm{SD}$ ). ${ }^{* *} \mathrm{P}<0.01$. (B) In situ hybridization for miR-200a in HCC tumor tissues and the corresponding non-tumor liver tissues was performed. Positive signals are shown as dark blue; nuclei are counterstained as red. a and b, Positive miR-200a staining in non-tumor liver tissues; $\mathrm{c}$ and d, negative staining in HCC tumor tissues. Panels b and d (x400) represent high magnifications of panels a and c (x100), respectively, from the highlighted areas. (C) miR-200a expression level in $7 \mathrm{HCC}$ cell lines and normal liver cell line LO2. (D) Expression levels of miR-200a in HepG2 and HCCLM3 cells treated by miR-200a mimics or inhibitors (50 nM) after $48 \mathrm{~h}$. Data shown are mean $\pm \mathrm{SD} .{ }^{*} \mathrm{P}<0.05,{ }^{* *} \mathrm{P}<0.01$.

3'UTR luciferase reporter plasmid construction. The 500-bp 3'UTR of human MACC1 complementary DNA was amplified by polymerase chain reaction with the following primers: forward, 5'-CCGCTCGAGCACCAGTAAAACAAGGAAC TTG-3' and reverse primer, 5'-GAATGCGGCCGCTTTACA GAAACAAATGCAATGTTAC-3'. Endonuclease restriction sites were incorporated in the primers to facilitate ligation into the luciferase reporter plasmid psiCHECK-2 vector (Promega, Madison, WI, USA). A psiCHECK-2 construct containing 3'UTR of MACC1 with a mutant seed sequence of miR-200a was also synthesized (Genepharma, Shanghai, China). All constructs were verified by DNA sequencing. HEK293 cells were plated in 96-well plates and then cotransfected with $100 \mathrm{ng}$ constructs with or without miR-200a. At $48 \mathrm{~h}$ after transfection, luciferase activity was detected using a dualluciferase reporter assay system (Promega) and normalized to Renilla activity.

Statistical analysis. The results are expressed as mean \pm standard deviation (SD), as appropriate. Comparisons of continuous data were analyzed by the Student $t$-test between two groups, whereas categorical data were analyzed by the Chi-square test.
Statistical analyses were performed using SPSS for Windows v.16.0 (SPSS, Inc., Chicago, IL, USA) and GraphPad Prism 5.0 (GraphPad Software, La Jolla, CA, USA). P $<0.05$ was considered to indicate a statistically significant result.

\section{Results}

miR-200a is frequently downregulated in hepatocellular carcinoma and HCC cell lines. We measured the expression of mature miR-200a in the human liver tissues and the corresponding HCCs ( $\mathrm{n}=115$ ) using quantitative RT-PCR (qRT-PCR) with the endogenous control (RNU6B). We found that miR-200a was markedly decreased in the HCCs $(\mathrm{P}<0.01 ;$ Fig. 1A). The overall expression level of miR-200a was decreased $\sim 2.36$-fold in the HCC samples. This result suggests that downregulation of miR-200a may be a frequent event in HCC.

To corroborate these findings, in situ hybridization analysis was performed in the HCCs and normal liver tissues using 5'- and 3'-dig-labeled LNA probes. In situ hybridization with LNA-modified anti-miR-200a probe also showed a decrease in the HCC samples. No signal was detected with the scrambled control demonstrating specificity of the probes (Fig. 1B). 


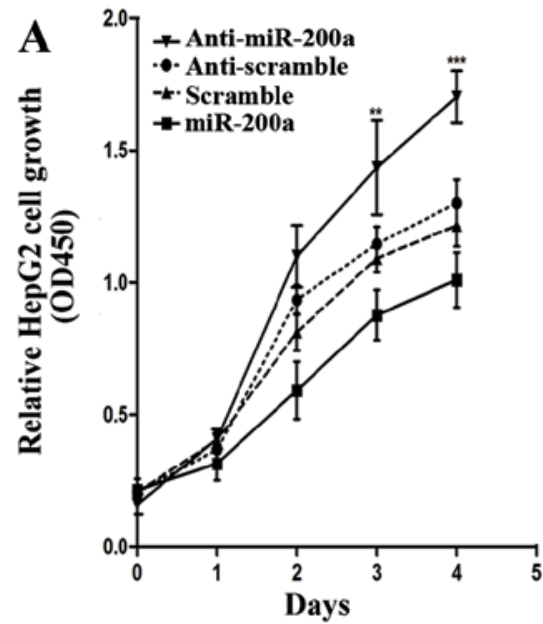

C
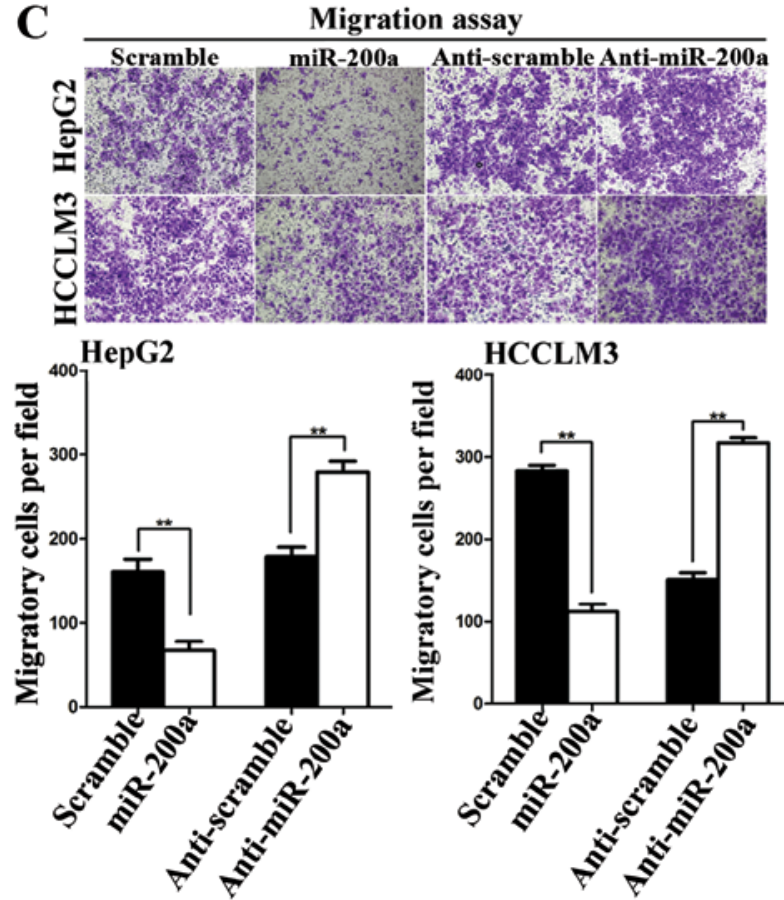

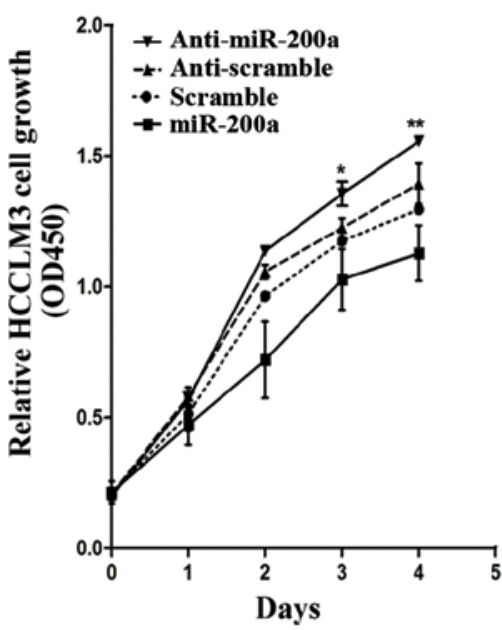

D

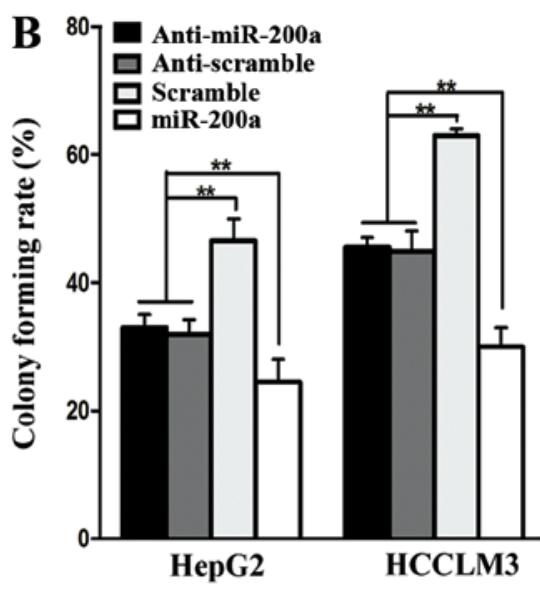

Invasion assay
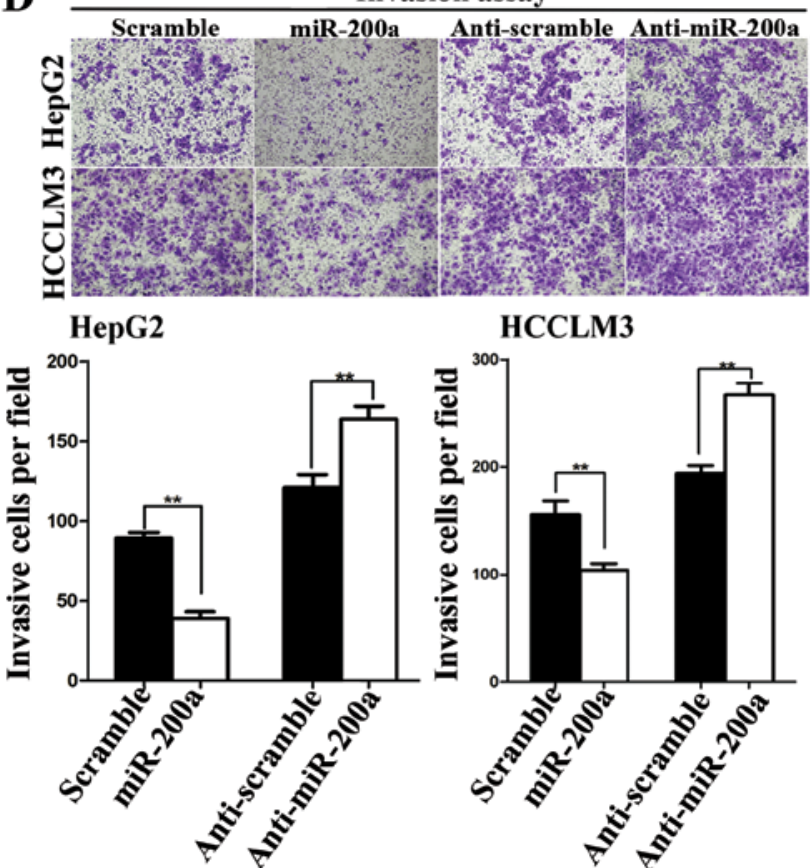

HCCLM3

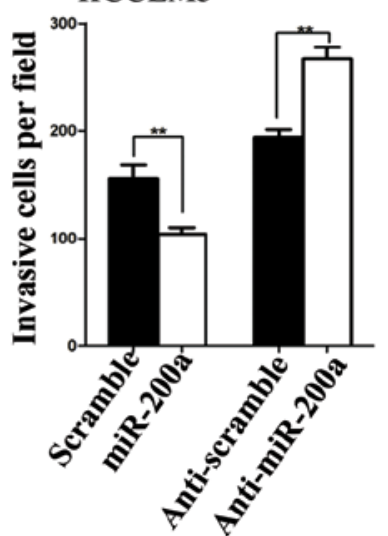

Figure 2. miR-200a inhibits tumor cell growth and metastasis in vitro. (A) The growth of HepG2 and HCCLM3 cells was determined by the CCK-8 assay. The growth of HepG2 and HCCLM3 cells transfected with the miR-200a mimics or the inhibitor against miR-200a expression was assessed. Data are presented as mean \pm SD from 3 independent experiments. (B) The colony formation assay was performed, and the number of colonies was counted and compared (C) Migration assay and (D) invasion assay of the HepG2 and HCCLM3 cells transfected with miR-200a mimics or inhibitors. The migration and invasion assays were measured by Transwell assays with Matrigel. ${ }^{*} \mathrm{P}<0.05,{ }^{* *} \mathrm{P}<0.01$ and ${ }^{* * * *} \mathrm{P}<0.001$.

We also investigated expression of miR-200a in 7 HCC cell lines and the normal liver cell line LO2. Except for the PLC cells, a low level of miR-200a was found in most of the HCC cell lines when compared with the expression in the LO2 cells. Among them, HCCLM3 and HepG2 cells were selected for subsequent investigation (Fig. 1C).

miR-200a inhibits tumor cell growth and metastasis in HCC. To investigate the effects of miR-200a on tumor biology, HCCLM3 and HepG2 cells were then transfected with miR-200a mimics and inhibitors or their respective controls and the efficiency of miR-200a and anti-miR-200a was validated (Fig. 1D).

To clarify the effect of miR-200a on tumor growth, cancer cell proliferation with CCK-8 further demonstrated that ectopic miR-200a markedly inhibited the cell viability of the HCCLM3 and HepG2 cells as compared with those transfected with the scramble control $(\mathrm{P}<0.01$; Fig. 2A). However, knockdown of miR-200a with miR-200a inhibitors restored the malignant cell proliferation $(\mathrm{P}<0.01$; Fig. $2 \mathrm{~A})$. In addition, ectopic miR-200a expression significantly suppressed colony forming rates both in the HCCLM3 and HepG 2 cells. Conversely, colony forming rates were increased by knocking down miR-200a expression ( $\mathrm{P}<0.01$; Fig. $2 \mathrm{~B}$ ). To elucidate the role of miR-200a in HCC metastasis, the effects of miR-200a on the migration and invasion of HCC cells were analyzed initially in vitro. Transwell assays showed that both the migratory and invasive abilities of the HCC cells were suppressed by $\mathrm{miR}-200 \mathrm{a}$ overexpression and the metastatic potential was enhanced when cellular miR-200a was neutralized by anti- 
Table I. Correlation between miR-200a expression and clinicopathological factors in $115 \mathrm{HCC}$ tumors.

\begin{tabular}{|c|c|c|c|}
\hline \multirow[b]{2}{*}{ Variable } & \multicolumn{2}{|c|}{ miR-200a expression } & \multirow[b]{2}{*}{ P-value ${ }^{a}$} \\
\hline & Low & High & \\
\hline \multicolumn{4}{|l|}{ Age (years) } \\
\hline$<50$ & 25 & 23 & 0.623 \\
\hline$\geq 50$ & 38 & 29 & \\
\hline \multicolumn{4}{|l|}{ Gender } \\
\hline Female & 9 & 5 & 0.446 \\
\hline Male & 54 & 47 & \\
\hline \multicolumn{4}{|c|}{ Preoperative tumor therapy } \\
\hline No & 46 & 41 & 0.468 \\
\hline Yes & 17 & 11 & \\
\hline \multicolumn{4}{|l|}{ Tumor size $(\mathrm{cm})$} \\
\hline$\leq 5$ & 34 & 33 & 0.304 \\
\hline$>5$ & 29 & 19 & \\
\hline \multicolumn{4}{|l|}{ Tumor number } \\
\hline Single & 35 & 30 & 0.818 \\
\hline Multiple & 28 & 22 & \\
\hline \multicolumn{4}{|c|}{ Tumor differentiation } \\
\hline Well + moderate & 34 & 29 & 0.847 \\
\hline Poor & 29 & 23 & \\
\hline \multicolumn{4}{|l|}{ Preoperative AFP } \\
\hline$\leq 400 \mathrm{ng} / \mathrm{ml}$ & 38 & 32 & 0.894 \\
\hline$>400 \mathrm{ng} / \mathrm{ml}$ & 25 & 20 & \\
\hline \multicolumn{4}{|l|}{ Vascular invasion } \\
\hline None & 34 & 33 & 0.304 \\
\hline Yes & 29 & 19 & \\
\hline
\end{tabular}

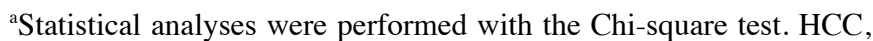
hepatocellular carcinoma, AFP, $\alpha$-fetoprotein.

miR-200a ( $\mathrm{P}<0.01$; Fig. $2 \mathrm{C}$ and $\mathrm{D})$. Collectively, the in vitro studies suggest the suppressive effects of miR-200a on tumor cell growth and metastasis in HCC.

miR-200a targets the 3'UTR of the MACC1 transcript and downregulates its expression. We then explored the molecular mechanism behind the inhibitory function of miR-200a on HCC growth and metastasis. Putative targets of miR-200a were predicted with TargetScan. Among these, MACC1 was chosen for further validation. The sequence of the predicted miR-200a binding sites and the MACC1 3'UTR segments containing the miR-200a complementary sequence are shown (Fig. 3A). qRT-PCR and western blotting further demonstrated that overexpression of miR-200a markedly suppressed the endogenous mRNA and protein levels of MACC1 respectively, whereas inhibition of miR-200a increased the expression of MACC1, in the HCCLM3 cells ( $\mathrm{P}<0.05$; Fig. 3B and C). The miR-200a or anti-miR-200a constructs were then cotransfected with the dual-luciferase reporter vector into HEK293 cells. A dual-luciferase reporter assay revealed that the cotransfection
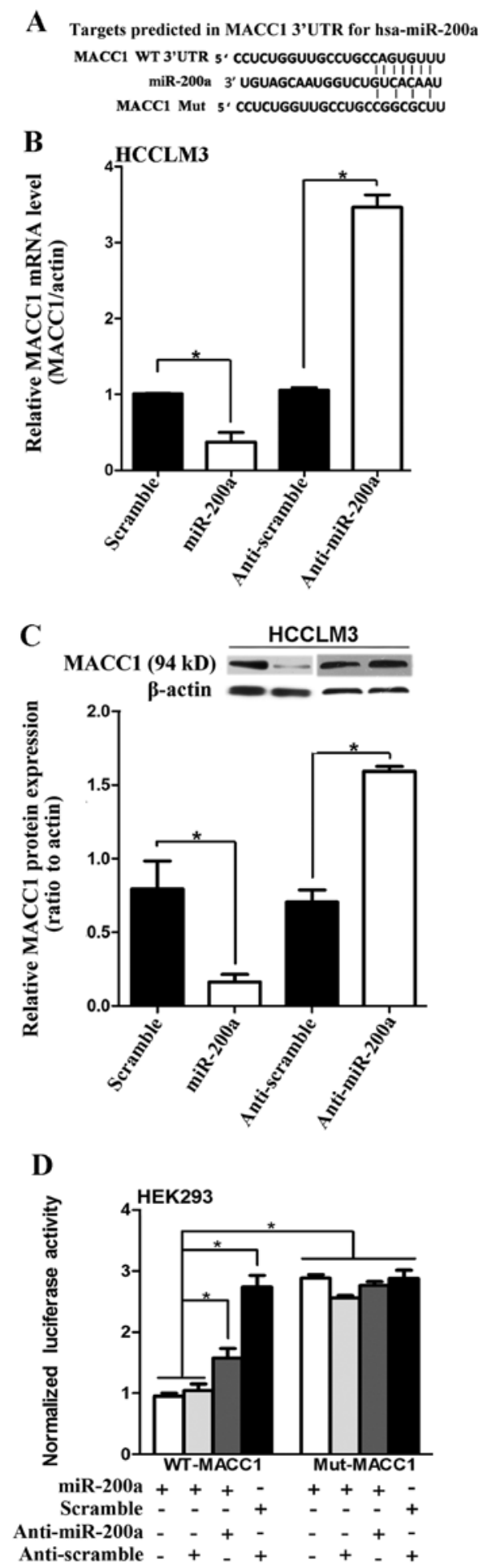

Figure 3. miR-200a targets the 3' untranslated region (3'UTR) of the MACC1 transcript and downregulates its expression. (A) The sequences of the predicted miR-200a binding site and the MACC1 3'UTR segments containing the wild-type or mutant binding site are shown. (B) qRT-PCR was performed to detect MACC1 mRNA levels in the transfected HCCLM3 cells. (C) Western blotting assay was used to detect the protein expression levels of MACC1 in the transiently transfected HCCLM3 cells. (D) Relative EGFP activity was analyzed after the wild-type or mutant 3'UTR reporter plasmids were cotransfected with miR-200a or anti-miR-200a in the HEK293 cells. The histogram shows the mean \pm SD of the normalized EGFP intensity from three independent experiments. ${ }^{*} \mathrm{P}<0.05$.

of miR-200a significantly inhibited the activity of the firefly luciferase reporter with wild-type 3'UTR of MACC1, whereas 

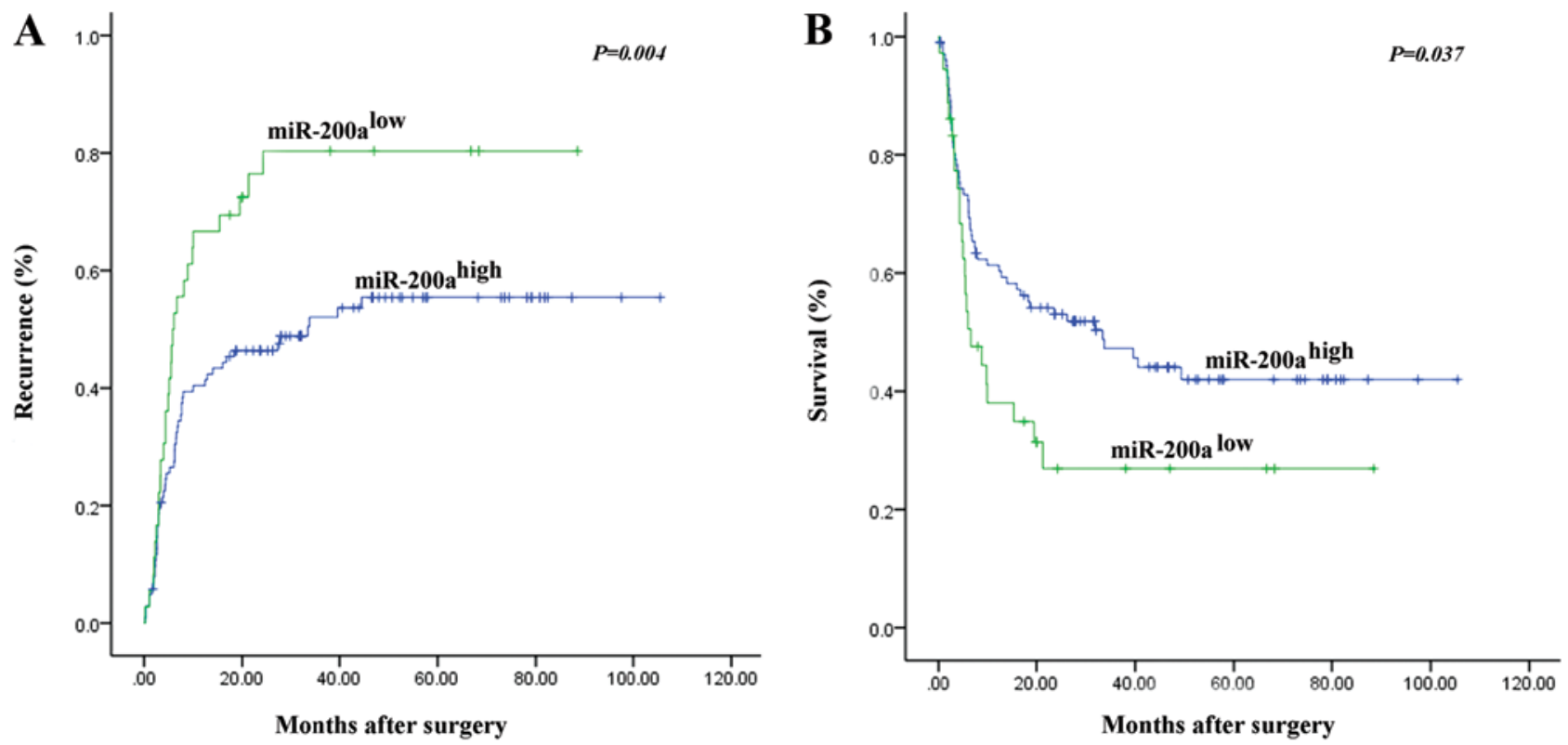

Figure 4. miR-200a expression is correlated with poor prognosis in hepatocellular carcinoma (HCC) patients following liver transplantation. Prognostic significance of miR-200a was assessed by Kaplan-Meier analysis and log-rank tests. The patients whose HCC tissue samples expressed low levels of miR-200a had a poor prognosis with respect to (A) cumulative recurrence and (B) overall survival.

Table II. miR-200a expression is an independent prognostic factor for HCC patients following LT.

\begin{tabular}{|c|c|c|c|c|}
\hline \multirow[b]{2}{*}{ Variable } & \multicolumn{2}{|c|}{ Cumulative recurrence } & \multicolumn{2}{|c|}{ Overall survival } \\
\hline & $\mathrm{HR}(95 \% \mathrm{CI})$ & P-value & $\mathrm{HR}(95 \% \mathrm{CI})$ & P-value \\
\hline \multicolumn{5}{|l|}{ Univariate analysis $^{\mathrm{a}}$} \\
\hline Age (year) ( $\geq 50$ vs. $<50$ ) & $0.546(0.355-0.841)$ & 0.006 & $0.808(0.521-1.253)$ & 0.341 \\
\hline Gender (male vs. female) & $2.161(0.834-5.095)$ & 0.117 & $2.654(0.970-7.261)$ & 0.057 \\
\hline Preoperative treatment (yes vs. no) & $1.196(0.747-1.916)$ & 0.457 & $1.025(0.617-1.703)$ & 0.924 \\
\hline Tumor size ( $>5$ vs. $\leq 5 \mathrm{~cm}$ ) & $3.934(2.527-6.123)$ & $<0.001$ & $3.070(1.960-4.809)$ & $<0.001$ \\
\hline Tumor number (multiple vs. single) & $2.595(1.637-4.114)$ & $<0.001$ & $2.356(1.468-3.779)$ & $<0.001$ \\
\hline $\begin{array}{l}\text { Tumor differentiation } \\
\text { (poor vs. well + moderate) }\end{array}$ & $1.485(0.947-2.265)$ & 0.066 & $1.043(0.672-1.620)$ & 0.851 \\
\hline $\begin{array}{l}\text { Preoperative AFP, } \mathrm{ng} / \mathrm{ml} \\
(>400 \text { vs. } \leq 400)\end{array}$ & $2.261(1.440-3.548)$ & $<0.001$ & $1.820(1.151-2.878)$ & 0.010 \\
\hline Vascular invasion (yes vs. none) & $2.995(1.959-4.580)$ & $<0.001$ & $2.310(1.491-3.579)$ & $<0.001$ \\
\hline miR-200a $\mathrm{a}^{\text {high }}$ vs. miR-200a $\mathrm{a}^{\text {low }}$ & $1.858(1.204-2.867)$ & 0.005 & $1.613(1.035-2.514)$ & 0.035 \\
\hline \multicolumn{5}{|l|}{ Multivariate analysis ${ }^{\mathrm{a}}$} \\
\hline Age (year) ( $\geq 50$ vs. $<50$ ) & - & 0.153 & - & - \\
\hline Tumor size ( $>5 \mathrm{vs} . \leq 5 \mathrm{~cm}$ ) & $3.350(2.123-5.288)$ & $<0.001$ & $2.719(1.725-4.286)$ & $<0.001$ \\
\hline Tumor number (multiple vs. single) & - & 0.141 & - & 0.150 \\
\hline $\begin{array}{l}\text { Preoperative AFP, } \mathrm{ng} / \mathrm{ml} \\
(>400 \text { vs. } \leq 400)\end{array}$ & $1.789(1.130-2.833)$ & 0.013 & $1.624(1.020-2.587)$ & 0.041 \\
\hline Vascular invasion (yes vs. none) & $2.267(1.474-3.485)$ & $<0.001$ & $1.853(1.192-2.880)$ & 0.006 \\
\hline miR-200a $a^{\text {high }}$ vs. miR-200a $a^{\text {low }}$ & $3.444(1.707-6.948)$ & 0.001 & $3.001(1.509-5.970)$ & 0.002 \\
\hline
\end{tabular}

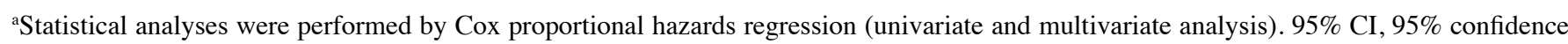
interval; HR, hazard ratio; HCC, hepatocellular carcinoma; LT, liver transplantation; AFP, $\alpha$-fetoprotein.

this effect was abrogated when the predicted 3'UTR binding site was mutated $(\mathrm{P}<0.05$; Fig. 3D). These data indicate that
miR-200a may negatively regulate MACC1 expression by directly targeting its 3 'UTR. 
Decreased miR-200a expression is correlated with poor prognosis in HCC patients following liver transplantation. To explore whether miR-200a may be a candidate biomarker for predicting the clinical outcome of HCC patients following LT, we examined the expression of miR-200a in $115 \mathrm{HCC}$ samples. Patients were segregated into high or low expression groups on the basis of receiver operating characteristic analysis. Following clinicopathological correlation analysis, the clinical characteristics not directly correlated with the expression of miR-200a included age, gender, tumor differentiation, vascular invasion, preoperative $\alpha$-fetoprotein (AFP) level, tumor number and tumor size (Table I).

Univariate analysis revealed that vascular invasion, preoperative serum AFP level (>400 ng/ml), multiple tumors and tumor size $(>5 \mathrm{~cm})$ and the expression level of miR-200a (miR-200a ${ }^{\text {high }}$ ) were predictors for overall survival (OS) and cumulative recurrence (Table II). HCC patients with low miR-200a expression had a significantly worse prognosis than those with high expression of miR-200a (Table II). The 1-, 3- and 5-year cumulative recurrence rates of miR-200a-low HCC were much higher than those of miR-200a-high HCC $(\mathrm{P}<0.001$; Fig. 4A). The 1-, 3- and 5-year overall survival rates were significantly lower for patients with miR-200a-low $\mathrm{HCC}$ than for patients with miR-200a-high HCC $(\mathrm{P}=0.037$; Fig. 4B).

Cox multivariate analysis (Table II) revealed that in addition to vascular invasion, a high preoperative AFP level $(>400 \mathrm{ng} / \mathrm{ml})$ and large tumor size $(>5 \mathrm{~cm})$, miR-200a overexpression in $\mathrm{HCC}$ was an independent prognostic factor for predicting tumor recurrence $(\mathrm{P}<0.001)$ and $\mathrm{OS}(\mathrm{P}<0.001)$ in HCC patients following LT.

\section{Discussion}

The development of early diagnostic and effective therapeutic strategies for $\mathrm{HCC}$ is an important issue for scientists and clinicians. Currently, there has been limited information regarding the differentiation states of HCC, although some information concerning HCC may be obtainable from surgical specimens. However, only 10-20\% of HCC patients are suitable for surgical operations, making it difficult to develop an effective and comprehensive therapeutic strategy for all HCC patients. In addition, a surgical operation is not indicated for treating HCC patients with metastatic tumors. A new biomarker of HCC progression may be helpful for early diagnosis or to determine a suitable solution for HCC therapy. Recently, miRNAs have become useful as prognostic and diagnostic clinical biomarkers. Determining whether the expression of specific miRNAs is correlated with the progressive state of HCC may be valuable for clinical applications.

miRNAs may function as critical regulators in the tumorigenesis of various human cancers, and loss of miR-200a expression has been reported in breast cancer, ovarian cancer and pancreatic cancer $(21,23-27)$. However, the expression pattern and its role in cell survival and metastasis of primary hepatocytes and the underlying mechanism have not been elucidated. In the present study, we identified that miR-200a was markedly downregulated in HCC and exerted a suppressive effect on tumor cell growth and metastasis. In addition, we found that miR-200a suppressed tumor growth and metastasis in $\mathrm{HCC}$ by directly targeting $\mathrm{MACC} 1$ through binding to a specific complementary site within its 3'UTR.

The miR-200 family has been linked to tumor progression and metastasis in several carcinomas. Members of the miR-200 family are downregulated during progression and migration of ovarian and breast cancer, as well as in bladder, pancreatic, prostate and esophageal carcinoma. Furthermore, in several of these entities, downregulation of miR-200 is associated with reduced overall survival and poor response to chemotherapy (28-35). In the present study, we found that HCC patients with low miR-200a expression had significantly worse prognosis than those with high expression of miR-200a.

In conclusion, our study revealed the inhibitory effect of miR-200a on MACC1 in HCC and partly elucidated a potential molecular mechanism by which miR-200a participates in tumor aggressiveness. These findings suggest that miR-200a may be recognized as a novel potential biomarker to predict the survival of patients with HCCs following LT.

\section{References}

1. Shiraha H, Yamamoto K and Namba M: Human hepatocyte carcinogenesis (Review). Int J Oncol 42: 1133-1138, 2013.

2. Finn RS: Emerging targeted strategies in advanced hepatocellular carcinoma. Semin Liver Dis 33 (Suppl 1): S11-S19, 2013.

3. Whittaker S, Marais R and Zhu AX: The role of signaling pathways in the development and treatment of hepatocellular carcinoma. Oncogene 29: 4989-5005, 2010.

4. Carr BI: Some new approaches to the management of hepatocellular carcinoma. Semin Oncol 39: 369-373, 2012.

5. Hamed O, Kimchi ET, Sehmbey M, Gusani NJ, Kaifi JT and Staveley-O'Carroll K: Impact of genetic targets on cancer therapy: hepatocellular cancer. Adv Exp Med Biol 779: 67-90, 2013.

6. Marrero JA: Multidisciplinary management of hepatocellular carcinoma: where are we today? Semin Liver Dis 33 (Suppl 1): S3-S10, 2013.

7. Alves RC, Alves D, Guz B, Matos C, Viana M, Harriz M, Terrabuio D, Kondo M, Gampel O and Polletti P: Advanced hepatocellular carcinoma. Review of targeted molecular drugs. Ann Hepatol 10: 21-27, 2011.

8. Herszényi L and Tulassay Z: Epidemiology of gastrointestinal and liver tumors. Eur Rev Med Pharmacol Sci 14: 249-258, 2010.

9. Hussain SA, Ferry DR, El-Gazzaz G, Mirza DF, James ND, McMaster P and Kerr DJ: Hepatocellular carcinoma. Ann Oncol 12: 161-172, 2001.

10. Tanaka M, Katayama F, Kato H, Tanaka H, Wang J, Qiao YL and Inoue $\mathrm{M}$ : Hepatitis $\mathrm{B}$ and $\mathrm{C}$ virus infection and hepatocellular carcinoma in China: a review of epidemiology and control measures. J Epidemiol 21: 401-416, 2011.

11. El-Serag HB: Hepatocellular carcinoma: an epidemiologic view. J Clin Gastroenterol 35 (5 Suppl 2): S72-S78, 2002.

12. Lai EC and Lau WY: The continuing challenge of hepatic cancer in Asia. Surgeon 3: 210-215, 2005.

13. Yuen MF, Hou JL and Chutaputti A; Asia Pacific Working Party on Prevention of Hepatocellular Carcinoma: Hepatocellular carcinoma in the Asia pacific region. J Gastroenterol Hepatol 24: 346-353, 2009.

14. Grossman EJ and Millis JM: Liver transplantation for non-hepatocellular carcinoma malignancy: Indications, limitations, and analysis of the current literature. Liver Transpl 16: 930-942, 2010.

15. Kaido T, Mori A, Ogura Y, Hata K, Yoshizawa A, Iida T and Uemoto S: Living donor liver transplantation for recurrent hepatocellular carcinoma after liver resection. Surgery 151: 55-60, 2012.

16. Ambros V, Bartel B, Bartel DP, Burge CB, Carrington JC, Chen X, Dreyfuss G, Eddy SR, Griffiths-Jones S, Marshall M, Matzke M, Ruvkun G and Tuschl T: A uniform system for microRNA annotation. RNA 9: 277-279, 2003.

17. Iorio MV and Croce CM: microRNA involvement in human cancer. Carcinogenesis 33: 1126-1133, 2012.

18. Schetter AJ and Harris CC: Alterations of microRNAs contribute to colon carcinogenesis. Semin Oncol 38: 734-742, 2011. 
19. Chen Y, Zhang L and Hao Q: Candidate microRNA biomarkers in human epithelial ovarian cancer: systematic review profiling studies and experimental validation. Cancer Cell Int 13: 86, 2013.

20. Feng B, Wang R and Chen LB: Review of miR-200b and cancer chemosensitivity. Biomed Pharmacother 66: 397-402, 2012.

21. Yao J, Zhou E, Wang Y, Xu F, Zhang D and Zhong D: microRNA-200a inhibits cell proliferation by targeting mitochondrial transcription factor A in breast cancer. DNA Cell Biol 33: 291-300, 2014.

22. Cong N, Du P, Zhang A, Shen F, Su J, Pu P, Wang T, Zjang J, Kang $\mathrm{C}$ and Zhang Q: Downregulated microRNA-200a promotes EMT and tumor growth through the wnt $/ \beta$-catenin pathway by targeting the E-cadherin repressors ZEB1/ZEB2 in gastric adenocarcinoma. Oncol Rep 29: 1579-1587, 2013.

23. Kobayashi M, Salomon C, Tapia J, Illanes SE, Mitchell MD and Rice GE: Ovarian cancer cell invasiveness is associated with discordant exosomal sequestration of Let-7 miRNA and miR-200. J Transl Med 12: 4, 2014.

24. Lam SS, Mak AS, Yam JW, Cheung AN, Ngan HY and Wong AS: Targeting estrogen-related receptor alpha inhibits epithelial-to-mesenchymal transition and stem cell properties of ovarian cancer cells. Mol Ther 22: 743-751, 2014.

25. Liu L, Zou J, Wang Q, Yin FQ, Zhang W and Li L: Novel microRNAs expression of patients with chemotherapy drugresistant and chemotherapy-sensitive epithelial ovarian cancer. Tumour Biol 35: 7713-7717, 2014.

26. Lu Y, Lu J, Li X, Zhu H, Fan X, Zhu S, Wang Y, Guo Q, Wang L, Huang Y, Zhu M and Wang Z: MiR-200a inhibits epithelialmesenchymal transition of pancreatic cancer stem cell. BMC Cancer 14: 85, 2014.

27. Sun Q, Zou X, Zhang T, Shen J, Yin Y and Xiang J: The role of miR-200a in vasculogenic mimicry and its clinical significance in ovarian cancer. Gynecol Oncol 132: 730-738, 2014.

28. Banyard J, Chung I, Wilson AM, Vetter G, Le Béchec A, Bielenberg DR and Zetter BR: Regulation of epithelial plasticity by miR-424 and miR-200 in a new prostate cancer metastasis model. Sci Rep 3: 3151, 2013
29. Cao Q, Lu K, Dai S, Hu Y and Fan W: Clinicopathological and prognostic implications of the miR-200 family in patients with epithelial ovarian cancer. Int J Clin Exp Pathol 7: 2392-2401, 2014.

30. Kolesnikoff N, Attema JL, Roslan S, Bert AG, Schwarz QP, Gregory PA and Goodall GJ: Specificity protein 1 (Sp1) maintains basal epithelial expression of the miR-200 family: implications for epithelial-mesenchymal transition. J Biol Chem 289: 11194-11205, 2014

31. Koutsaki M, Spandidos DA and Zaravinos A: Epithelialmesenchymal transition-associated miRNAs in ovarian carcinoma, with highlight on the miR-200 family: prognostic value and prospective role in ovarian cancer therapeutics. Cancer Lett 351: 173-181, 2014.

32. Song F1, Yang D, Liu B, Guo Y, Zheng H, Li L, Wang T, Yu J, Zhao Y, Niu R, Liang H, Winkler $\mathrm{H}$, Zhang W, Hao $\mathrm{X}$ and Chen K: Integrated microRNA network analyses identify a poor-prognosis subtype of gastric cancer characterized by the miR-200 family. Clin Cancer Res 20: 878-889, 2014

33. Truong HH, Xiong J, Ghotra VP, Nirmala E, Haazen L, Le Dévédec SE, Balcioğlu HE, He S, Snaar-Jagalska BE, Vreugdenhil E, Meerman JH, van de Water B and Danen EH: $\beta 1$ integrin inhibition elicits a prometastatic switch through the TGF $\beta$-miR-200-ZEB network in E-cadherin-positive triplenegative breast cancer. Sci Signal 7: ra15, 2014.

34. Wang CH, Chen CL, More SV, Hsiao PW, Hung WC and Li WS The tetraindole SK228 reverses the epithelial-to-mesenchymal transition of breast cancer cells by up-regulating members of the miR-200 family. PLoS One 9: e101088, 2014.

35. Wang CM, Liu R, Wang L, Nascimento L, Brennan VC and Yang WH: SUMOylation of FOXM1B alters its transcriptional activity on regulation of miR-200 family and JNK1 in MCF7 human breast cancer cells. Int J Mol Sci 15: 10233-10251, 2014. 